\title{
ON THE ASYMPTOTIC SOLUTIONS OF ORDINARY DIFFERENTIAL EQUATIONS, WITH REFERENCE TO THE STOKES' PHENOMENON ABOUT A SINGULAR POINT*
}

\author{
BY \\ RUDOLPH E. LANGER
}

1. Introduction. If in an ordinary differential equation of the second order, written in the form

$$
\frac{d^{2} w}{d s^{2}}+\{\lambda \psi(s)+\tau(\lambda, s)\} w=0,
$$

$\lambda$ represents a large parameter, it is frequently of importance to know the character of the asymptotic dependence of the solutions upon this parameter and upon the variable $s$. The literature of differential equations records many investigations of this matter. If the variable $s$ ranges over a real interval $R_{\boldsymbol{s}}$, or more generally over a region $R_{s}$ of the complex plane, on which the coefficients $\psi(s)$ and $\tau(\lambda, s)$ are bounded and the former is bounded from zero, there exist continuous forms composed of elementary functions of which each represents a solution over the entire region $R_{s} . \dagger$ On the other hand, if the coefficient $\psi(s)$ becomes zero at some point of $R_{s}$ the situation is more intricate. To represent one and the same solution an asymptotic form must then be constructed of other than elementary functions, or in the alternative, i.e., if it is to be of the simpler type, it is subject to the Stokes' phenomenon. The latter requires that the form change abruptly in a specifiable but intricate way as certain frontiers both in the $s$ and $\lambda$ planes are traversed. The theory of these asymptotic solutions, it being still supposed that the coefficient $\tau(\lambda, s)$ is bounded as to $s$, has been given, $\ddagger$ and applies to a number of standard differential equations. The list includes among others the equations for the Bessel functions, $\S$ the Hermite or Weber functions, $\|$ the Mathieu func-

* Presented to the Society, September 6, 1934; received by the editors July 6, 1934.

$\dagger$ With certain conditions when $R_{8}$ is infinite.

$\ddagger$ Langer, R. E., On the asymptotic solutions of ordinary differential equations, etc., these Transactions, vol. 33 (1931), pp. 23-64, vol. 34 (1932), pp. 447-480, vol. 36 (1934), pp. 90-106. For a descriptive account, literature and applications cf. also Langer, R. E., The asymptotic solutions of ordinary linear differential equations of the second order, with special reference to the Stokes' phenomenon, Bulletin of the American Mathematical Society, vol. 40 (1934), pp. 545-582.

§ Langer, R. E., loc. cit.

|| Schwid, N., On the Asymptotic Forms of the Hermite and Weber Functions, Thesis (1934) University of Wisconsin; see these Transactions, vol. 37 (1935), pp. 339-362. 
tions, ${ }^{*}$ the Laguerre functions and certain of the confluent forms of the hypergeometric functions.

The incidence of the Stokes' phenomenon has been associated with the vanishing of the coefficient $\psi(s)$, and its quantitative aspects with the order to which that coefficient becomes zero. It is shown in the present paper that this phenomenon is engendered also by an infinity in either of the coefficients, and is quantitatively dependent upon the structure of that infinity. More generally $\tau(\lambda, s)$ and $\psi(s)$ may, simultaneously or singly, become respectively infinite and either infinite or zero, and it is this inclusive situation which is discussed in the present investigation. Specifically the coefficient $\tau(\lambda, s)$ is admitted to have a pole of the first or second order, while $\psi(s)$ is taken to contain as a factor the quantity $\left(s-s_{0}\right)^{\nu}$, with $\nu$ a (any) real constant exceeding -2 . A number of standard differential equations may be brought under this general type, in particular the equations for the ordinary or the associated Legendre functions, for the Laguerre functions, and for the Mathieu functions of higher order. $\dagger$ The asymptotic representations of such functions with variable and parameter complex, may accordingly be obtained by suitable specializations of the formulas with which the present discussion culminates.

2. The normal form of the given differential equation. Let the differential equation, reduced by the usual removal of the term of the first order, be of the form (1), with $\lambda$ a sufficiently large value which may be complex. This equation is to be considered in a domain $R_{s}$ which is simply connected; which may be finite or infinite; and which contains the point designated below by $s_{0}$. The equation, the domain, and the admitted range of parameter values are aggregately to fulfill a set of hypotheses which will be numbered from (i) to (vi) and which will be enunciated at appropriate points in the sequel. The initial pair are as follows:

(i) Within $R_{s}$ the coefficient $\psi(s)$ is of the form

$$
\psi(s) \equiv\left(s-s_{0}\right)^{\nu} \psi_{1}(s),
$$

with $\nu>-2$, and with $\psi_{1}(s)$ a non-vanishing single-valued analytic function.

(ii) Within $R_{s}$ the coefficient $\tau(\lambda, s)$ is of the form

$$
\tau(\lambda, s) \equiv \frac{A_{1}}{\left(s-s_{0}\right)^{2}}+\frac{B_{1}}{s-s_{0}}+C_{1}(\lambda, s),
$$

with $A_{1}$ and $B_{1}$ any constants, and $C_{1}(\lambda, s)$ an analytic function which on any finite portion of $R_{s}$ is bounded uniformly with respect to $\lambda$.

* Langer, R. E., The solutions of the Mathieu equation, etc., these Transactions, vol. 36 (1934), pp. $637-695$.

$\dagger$ Cf. Humbert, P., Proceedings of the Edinburgh Mathematical Society, 1921-22, p. 27. 
In the product $\lambda \psi(s)$ the constant factors may be, and will be, taken to be distributed between $\lambda$ and $\psi(s)$ so that the latter expands in the form

$$
\psi(s) \equiv\left(s-s_{0}\right)^{\nu}\left\{1+\alpha_{1}\left(s-s_{0}\right)+\alpha_{2}\left(s-s_{0}\right)^{2}+\cdots\right\} .
$$

If in this formula and in that for $\tau(\lambda, s)$ above, the constants $\alpha_{1}$ and $B_{1}$ are both zero the differential equation will be defined to be normal. In this case at most a change of origin and lettering may be made to give it the form

$$
\frac{d^{2} u}{d z^{2}}+\left\{\rho^{2} \phi^{2}(z)+\frac{\frac{1}{4}-A^{2}}{z^{2}}+\chi(\rho, z)\right\} u=0 .
$$

In the contrary case the equation may always be normalized by the substitutions

$$
\left(s-s_{0}\right)=\frac{z^{2}}{4}, \quad w=z^{1 / 2} u,
$$

the symbols of the form (1) leading then to those of (2) as is shown thus:

$$
\begin{gathered}
\rho=\lambda^{1 / 2} / 2^{\nu+1}, \quad A^{2}=1-4 A_{1}, \\
\chi(\rho, z)=B_{1}-\frac{z^{2}}{4} C_{1}(\lambda, s), \\
\phi^{2}(z)=z^{1 / \mu-2} \psi_{1}(s), \quad \mu=\frac{1}{2(\nu+2)} .
\end{gathered}
$$

The form (2) will be made basic for the discussion which follows. The facts to be especially noted at this point are the following. First, the constant $\mu$ is always real and positive (not zero), but is not otherwise restricted as to magnitude. Second, $A^{2}$ is an unrestricted constant real or complex. For definiteness the designation $A$ will be reserved for that root of $A^{2}$ for which (unless it is zero)

$$
-\pi / 2<\arg A \leqq \pi / 2 .
$$

Third, if the constant $\nu$ is not an even integer, the region $R_{s}$ must be considered as a Riemann surface with a branch point at $s_{0}$ if unique values are to be assigned to $\psi^{1 / 2}(s)$. The relation between $s$ and $z$ maps this region upon a domain, to be denoted by $R_{z}$, which is in general also a Riemann surface (or a part of such). Upon this surface, whose branch point is at the origin, the functions $\phi(z)$ and $\chi(\rho, z)$ are single-valued and analytic, and on any finite portion of it $\chi(\rho, z)$ is bounded uniformly with respect to the parameter $\rho$. Finally, the symbol $\phi(z)$ will be understood to represent that root of $\phi^{2}(z)$ which is determined by the relation

$$
\lim _{z \rightarrow 0}\left\{\phi(z) / z^{1 /(2 \mu)-1}\right\}=1 .
$$

3. The "related" differential equation. The formulas 


$$
\Phi=\int_{0}^{z} \phi(z) d z, \quad \xi=\rho \Phi
$$

may be looked upon as defining the complex variables $\Phi$ and $\xi$, whose domains of variation are to be designated respectively by $R_{\Phi}$ and $R_{\xi}$. Each of these domains in general lies upon a Riemann surface with branch point at the origin, and consists of sheets in number finite or infinite as the character of the constant $\mu$ may determine. In a neighborhood of the origin the correspondence between points $\Phi$ and $z$ is of the "one to one" type. It is to be an hypothesis that this is so for the entire domains considered, i.e.,

(iii) The region $R_{z}$ is such that the correspondence of points of the Riemann surfaces $R_{\Phi}$ and $R_{z}$ is unique.

It will be observed that the domains $R_{\xi}$ and $R_{\Phi}$ differ only in scale and orientation, the respective factors depending upon $\rho$. In particular, it is to be noted that since some neighborhood of $z=0$ lies in $R_{z}$, therefore the region $|\xi| \leqq N$, with any fixed constant $N$, lies entirely within $R_{\xi}$ when $|\rho|$ is sufficiently large. Also as arg $\rho$ varies the domain $R_{\xi}$ rotates, so that any locus in $\xi$, fixed relatively to $R_{\xi}$, has an image locus in $R_{z}$ which varies with $\rho$.

It is a consequence of the hypotheses that both $\phi$ and $\Phi$ are finite and different from zero except possibly at the origin. Hence the function

$$
\Psi(z) \equiv \phi^{-1 / 2}(z) \Phi^{1 / 2-\mu}(z),
$$

which is indeterminate at the origin, may be taken as so defined there that it and its reciprocal are analytic over the entire region $R_{z}$.

Consider the function

$$
y(z) \equiv \Psi(z) \xi^{\mu} C_{\beta}(\xi),
$$

in which $C_{\beta}$ represents any cylinder function of the order $\beta$, the latter being as yet unspecified. It is found that this function solves the differential equation

$$
y^{\prime \prime}+\left\{\rho^{2} \phi^{2}+\left(\mu^{2}-\beta^{2}\right) \frac{\phi^{2}}{\Phi^{2}}-\frac{\Psi^{\prime \prime}}{\Psi}\right\} y=0 .
$$

Since $\phi^{2} / \Phi^{2}$ differs from $1 /\left(4 \mu^{2} z^{2}\right)$ only by an analytic function, the choice

$$
\beta=2 \mu A
$$

reduces the equation to the form

$$
\frac{d^{2} y}{d z^{2}}+\left\{\rho^{2} \phi^{2}(z)+\frac{\frac{1}{4}-A^{2}}{z^{2}}+\omega(z)\right\} y=0,
$$

with $\omega(z)$ a coefficient which is analytic throughout $R_{z}$. The differential equa- 
tion (7), in so far as its more immediately essential features are concerned, is identical with the given equation (2). It will therefore be referred to as the related equation. It is explicitly and generally solved by the formula (5). The index $\beta$ may evidently be zero or any complex constant subject to

$$
-\pi / 2<\arg \beta \leqq \pi / 2 \text {. }
$$

4. The solutions of the related equation. If the cylinder function in formula (5) is chosen in turn as the Bessel function of the first and second kind, the solutions obtained are

$$
\begin{aligned}
& y_{1}(z) \equiv \Psi(z) \xi^{\mu} J_{\beta}(\xi), \\
& y_{2}(z) \equiv \Psi(z) \xi^{\mu} Y_{\beta}(\xi) .
\end{aligned}
$$

These functions are linearly independent, their Wronskian having the value

$$
W\left(y_{1}, y_{2}\right)=\frac{-2}{\pi} \rho^{2 \mu},
$$

and near $\xi=0$ they are of the forms

$$
\begin{aligned}
& y_{1}(z)=\xi^{\mu+\beta} O(1), \\
& y_{2}(z)= \begin{cases}\xi^{\mu-\beta} O(1), & \text { if } \beta \neq 0, \\
\xi^{\mu} \log \xi O(1), & \text { if } \beta=0,\end{cases}
\end{aligned}
$$

with $O(1)$ signifying in each case a bounded function.

From the formulas (9) it may be seen, since $\xi=O\left(z^{1 /\left(\mu_{\mu}\right)}\right)$, that by an arbitrary approach to the origin on which $\arg z$ remains bounded the solution $y_{1}(z)$ invariably approaches zero. Moreover, if $\Re(A)>0, y_{1}(z)$ is determined uniquely (except for a constant factor) as the solution which vanishes at $z=0$ to a higher order than any other. By a similar approach to the origin $y_{2}(z)$ either also approaches zero or else becomes infinite according as the real component of the constant $A$ is less than or greater than $\frac{1}{2}$. Clearly the same necessarily follows for any solution which is linearly independent of $y_{1}$.

In virtue of the relation

$$
\frac{d \xi}{d z}=\frac{\rho^{2 \mu}}{\Psi^{2}(z)} \xi^{1-2 \mu}
$$

which is readily derived from (3) and (4), the differentiation of the formulas (9) with respect to $z$ leads to

$$
\begin{aligned}
& y_{1}^{\prime}(z)=\rho^{2 \mu \xi^{-\mu+\beta}} O(1), \\
& y_{2}^{\prime}(z)= \begin{cases}\rho^{2 \mu} \xi^{-\mu-\beta} O(1), & \text { if } \beta \neq 0, \\
\rho^{2 \mu} \xi^{-\mu} \log \xi O(1), & \text { if } \beta=0 .\end{cases}
\end{aligned}
$$


The solutions (8) are especially adapted to such considerations as involve primarily values of $\xi$ near the origin. On the other hand, when large values of $\xi$ are in question the solutions obtained by using the Bessel functions of the third kind in formula (5) are of advantage. The formulas

$$
\begin{aligned}
y_{2 m+1,1}(z) & \equiv y_{2 m, 1}(z), \\
y_{2 m, 1}(z) & \equiv\left(\frac{\pi}{2}\right)^{1 / 2} e^{(\beta+2 m+1 / 2) \pi i / 2} \Psi(z) \xi^{\mu} H_{\beta}^{(1)}\left(\xi e^{-2 m \pi i}\right), \\
y_{2 m-1,2}(z) & \equiv y_{2 m, 2}(z), \\
y_{2 m, 2}(z) & \equiv\left(\frac{\pi}{2}\right)^{1 / 2} e^{-(\beta+2 m+1 / 2) \pi i / 2} \Psi(z) \xi^{\mu} H_{\beta}^{(2)}\left(\xi e^{-2 m \pi i}\right)
\end{aligned}
$$

associate a pair of such solutions $y_{k, j}(z), j=1,2$, with each integral index $k$. The members of any pair are linearly independent, their Wronskian being

$$
W\left(y_{k, 1}, y_{k, 2}\right)=2 i \rho^{2 \mu},
$$

and each is invariably independent of $y_{1}(z)$. Near the origin, therefore (if $\arg z$ is bounded),

$$
y_{k, j}(z)= \begin{cases}\xi^{\mu-\beta} O(1), & \text { if } \beta \neq 0, \\ \xi^{\mu} \log \xi O(1), & \text { if } \beta=0, \quad j=1,2,\end{cases}
$$

and

$$
y_{k, j}^{\prime}(z)= \begin{cases}\rho^{2 \mu} \xi^{-\mu-\beta} O(1), & \text { if } \beta \neq 0 \\ \rho^{2 \mu} \xi^{-\mu} \log \xi O(1), & \text { if } \beta=0\end{cases}
$$

Let $\epsilon$ be chosen as any fixed sufficiently small but positive constant. Then the domain $R_{\xi}$ is sub-divided into a set of overlapping sub-regions $\Xi^{(l)}$, $l=0, \pm 1, \pm 2, \cdots$, by the relations

$$
\Xi^{(l)}:(l-1+\epsilon) \pi \leqq \arg \xi \leqq(l+1-\epsilon) \pi .
$$

The corresponding sub-regions of $R_{z}$ may without confusion be designated by the same symbol. Since the sub-region $\Xi^{(l)}$ on $R_{\xi}$ is fixed relative to $R_{\xi}$, the sub-region $\Xi^{(l)}$ in $R_{z}$ will be dependent upon and variable with $\rho$.

When $\xi$ is numerically sufficiently large, a condition which is to be indicated briefly by the symbolism $|\xi|>N$, the solutions of the differential equation (7) admit of asymptotic representations. The solutions (11) were especially chosen so that their representations are peculiarly simple when $\xi$ lies in suitably associated sub-regions (13), i.e., specifically 


$$
\begin{aligned}
& y_{2 m, 1}(z)=\Psi(z) \xi^{\mu-1 / 2} e^{i \xi}\left\{1+O\left(\frac{1}{\xi}\right)\right\}, \text { for } \xi \text { in } \Xi^{(2 m)} \text { and } \Xi^{(2 m+1)}, \\
& y_{2 m, 2}(z)=\Psi(z) \xi^{\xi-1 / 2} e^{-i \xi}\left\{1+O\left(\frac{1}{\xi}\right)\right\}, \text { for } \xi \text { in } \Xi^{(2 m-1)} \text { and } \Xi^{(2 m)} .
\end{aligned}
$$

The differentiation of these forms is permissible and yields

$$
\begin{aligned}
& y_{2 m, 1}^{\prime}(z)=\frac{i \rho^{2 \mu}}{\Psi(z)} \xi^{-\mu+1 / 2} e^{i \xi}\left\{1+O\left(\frac{1}{\xi}\right)\right\}, \quad \text { for } \xi \text { in } \Xi^{(2 m)} \text { and } \Xi^{(2 m+1)}, \\
& y_{2 m, 2}^{\prime}(z)=\frac{-i \rho^{2 \mu}}{\Psi(z)} \xi^{-\mu+1 / 2} e^{-i \xi}\left\{1+O\left(\frac{1}{\xi}\right)\right\}, \text { for } \xi \text { in } \Xi^{(2 m-1)} \text { and } \Xi^{(2 m)} .
\end{aligned}
$$

A solution of the differential equation is generally found to become exponentially infinite with $|\Im(\xi)|$. When such is the case the solution will be described as of the dominant type for the range of values $\xi$ concerned. Exceptionally, however, the solution approaches zero under the stated circumstances. In that case it will be described as of the sub-dominant type. From the formulas (14) it is evident that in the domain common to the subregions $\Xi^{(2 m)}$ and $\Xi^{(2 m+1)}$ the associated solution $y_{2 m, 1}(z)$ is sub-dominant, since in this domain $\Im(\xi) \rightarrow+\infty$ with $|\xi|$. In the remaining parts of the specified sub-regions it is dominant. Likewise the solution $y_{2 m, 2}(z)$ is subdominant in the domain common to $\Xi^{(2 m-1)}$ and $\Xi^{(2 m)}$, since there $\Im(\xi) \rightarrow-\infty$, and in the remaining parts of these sub-regions is dominant. These facts may be briefly though loosely stated thus: For any index $k$, $y_{k, 1}(z)$ is sub-dominant in the "upper half" and dominant in the "lower half" of $\Xi^{(k)}$, while $y_{k, 2}(z)$ is sub-dominant in the "lower half" and dominant in the "upper half" of $\Xi^{(k)}$. Since any solution which is linearly independent of the sub-dominant one on a given range must include a component of the dominant one, it must evidently itself be of the dominant type. It follows that the solutions (11) are in fact determined uniquely (except for constant factors) by their properties of sub-dominance as described.

The formulas (14), specifying as they do certain sub-regions (13), in general cease to be valid when $\xi$ passes out of these regions. This is the Stokes' phenomenon. To obtain the representations when $\xi$ lies in some other nonassociated sub-region, say $\Xi^{(h)}$, it is, however, merely necessary to express the given solutions in terms of the solutions $y_{h, j}(z)$, and to utilize the formulas (14) for the latter. The relation between distinct pairs of solutions (11) which is thus brought into question is obtainable from the known relation 
between the Bessel functions involved.* It is found in this way that when $|\xi|>N$, then

$$
\begin{aligned}
y_{2 m, j}(z)= & \Psi(z) \xi^{\mu-1 / 2}\left\{c_{j, 1}^{(m)} e^{i \xi}\left[1+O\left(\frac{1}{\xi}\right)\right]\right. \\
& \left.+c_{j, 2}^{(m)} e^{-i \xi}\left[1+O\left(\frac{1}{\xi}\right)\right]\right\},
\end{aligned}
$$

with coefficients as follows:

$$
\begin{aligned}
& \left.\begin{array}{rl}
c_{1,1}^{(m)} & =(-1)^{m-s+1} \frac{\sin (2 s-2 m-1) \beta \pi}{\sin \beta \pi} \\
c_{2,1}^{(m)} & =(-1)^{m-s+1} \frac{i \sin (2 s-2 m) \beta \pi}{\sin \beta \pi}
\end{array}\right\} \text { for } \xi \text { in } \Xi^{(2 s-1)} \text { or } \Xi^{(2 s)}, \\
& \left.\begin{array}{c}
c_{1,2}^{(m)}=(-1)^{m-s+1} \frac{i \sin (2 s-2 m) \beta \pi}{\sin \beta \pi} \\
c_{2,2}^{(m)}=(-1)^{m-s} \frac{\sin (2 s-2 m+1) \beta \pi}{\sin \beta \pi}
\end{array}\right\} \text { for } \xi \text { in } \Xi^{(2 s)} \text { or } \Xi^{(2 s+1)} .
\end{aligned}
$$

The asymptotic form of the solution $y_{1}(z)$ is analogously given by the formulas $\dagger$

$$
y_{1}(z)=\Psi(z) \xi^{\mu-1 / 2}\left\{c_{1} e^{i \xi}\left[1+O\left(\frac{1}{\xi}\right)\right]+c_{2} e^{-i \xi}\left[1+O\left(\frac{1}{\xi}\right)\right]\right\},
$$

and

$$
y_{1}^{\prime}(z)=\frac{i \rho^{2 \mu \xi^{-\mu+1 / 2}}}{\Psi(z)}\left\{c_{1} e^{i \xi}\left[1+O\left(\frac{1}{\xi}\right)\right]-c_{2} e^{-i \xi}\left[1+O\left(\frac{1}{\xi}\right)\right]\right\},
$$

with coefficients

$$
\begin{aligned}
& c_{1}=(2 \pi)^{-1 / 2} e^{(2 s-1 / 2)(\beta+1 / 2) \pi i}, \text { for } \xi \text { in } \Xi^{(28-1)} \text { or } \Xi^{(2 s)}, \\
& c_{2}=(2 \pi)^{-1 / 2} e^{(2 s+1 / 2)(\beta+1 / 2) \pi i}, \text { for } \xi \text { in } \Xi^{(28)} \text { or } \Xi^{(2 s+1)} .
\end{aligned}
$$

Since the coefficients (18) are different from zero for every index, the solution $y_{1}(z)$ is seen to be of the dominant type in both the upper and the lower "half" of any and every sub-region (13).

For subsequent use it may be observed that the expression

$$
\left\{y_{p}(z) y_{q}\left(z_{1}\right)-y_{q}(z) y_{p}\left(z_{1}\right)\right\} / W\left(y_{p}, y_{q}\right),
$$

lcoked upon as a ratio of determinants, is obviously independent of the choice of the solutions $y_{p}$ and $y_{q}$. It follows that the expression

* Watson, G. M., A Treatise on the Theory of Bessel Functions, Cambridge, 1922, p. 75.

† Watson, loc. cit., p. 202. 
(19a) $Q\left(z, z_{1}\right) \equiv \frac{\pi}{2}\left\{y_{1}(z) y_{2}\left(z_{1}\right)-y_{2}(z) y_{1}\left(z_{1}\right)\right\} \frac{\Psi^{3}\left(z_{1}\right)}{\Psi(z)}\left\{\chi\left(\rho, z_{1}\right)-\omega\left(z_{1}\right)\right\} \xi_{1}^{2 \mu-1}$,

which is to be used below, may equally well be written

(19b) $Q\left(z, z_{1}\right) \equiv \frac{i}{2}\left\{y_{k, 1}(z) y_{k, 2}\left(z_{1}\right)-y_{k, 2}(z) y_{k, 1}\left(z_{1}\right)\right\} \frac{\Psi^{3}\left(z_{1}\right)}{\Psi(z)}\left\{\chi\left(\rho, z_{1}\right)-\omega\left(z_{1}\right)\right\} \xi_{1}^{2 \mu-1}$,

with any choice of the index $k$.

5. The solution $u_{1}(z)$ when $|\xi| \leqq N$. It is convenient for the considerations at hand to designate briefly as a " $\Gamma$ curve" any ordinary curve upon which as seen in $R_{\xi}$ the ordinate varies monotonically with the arc length, and upon which the variation of arg $\xi$ remains below a (some) finite bound independent of the particular curve. Inasmuch as this description is relative to $R_{\xi}$ the curves in question as seen in $R_{z}$ depend, of course, upon $\rho$. The following, which is to be made an hypothesis, therefore essentially applies to a conjunction of the admitted range of values $\rho$ with the configuration of the region $R_{z}$.

(iv) The region $R_{z}$ is such that for any (every) admitted value of $\rho$ each point may be connected with the origin by some " $\Gamma$ curve" which lies entirely in the region.

Let the function $\theta(\rho, z)$ be defined by the formula

$$
\theta(\rho, z) \equiv \chi(\rho, z)-\omega(z) .
$$

It is clearly analytic in $z$ and bounded uniformly as to $\rho$ in any finite portion of $R_{z}$. Since the differential equation (2) may be written in the form

$$
\frac{d^{2} u}{d z^{2}}+\left\{\rho^{2} \phi^{2}(z)+\frac{\frac{1}{4}-A^{2}}{z^{2}}+\omega(z)\right\} u=-\theta(\rho, z) u,
$$

the left member of which is identical in structure with that of the equation (7), it follows that the formula

$$
u(z)=y(z)+\frac{\pi}{2 \rho^{2 \mu}} \int_{z_{0}}^{z}\left\{y_{1}(z) y_{2}\left(z_{1}\right)-y_{2}(z) y_{1}\left(z_{1}\right)\right\} \theta\left(\rho, z_{1}\right) u\left(z_{1}\right) d z_{1},
$$

with any limit $z_{0}$ independent of $z$, relates a solution of equation (2) with any solution $y(z)$ of the equation (7). This relationship will be indicated consistently by the use of similar subscripts.

The differentiation of (20) yields the associated formula

$\left(20^{\prime}\right) u^{\prime}(z)=y^{\prime}(z)+\frac{\pi}{2 \rho^{2 \mu}} \int_{z_{0}}^{z}\left\{y_{1}^{\prime}(z) y_{2}\left(z_{1}\right)-y_{2}^{\prime}(z) y_{1}\left(z_{1}\right)\right\} \theta\left(\rho, z_{1}\right) u\left(z_{1}\right) d z_{1}$. 
The path of integration, to be inferred from (20) or $\left(20^{\prime}\right)$ as in $R_{z}$, may with greater convenience be considered in the region $R_{\xi}$, the transformation being facilitated by the use of formula (10). If the fixed limit of integration is chosen as the origin, and this is to be the case in the present and the following section, the path may and will be chosen as a curve of the type $\Gamma$. With the introduction of the abbreviations

$$
Y_{1}(z) \equiv \frac{e^{-i \xi}}{\Psi(z)} y_{1}(z), \quad U_{1}(z) \equiv \frac{e^{-i \xi}}{\Psi(z)} u_{1}(z),
$$

the relation (20) may then be written in the form

$$
U_{1}(z)=Y_{1}(z)+\frac{1}{\rho^{4 \mu}} \int_{\Gamma} Q\left(z, z_{1}\right) e^{-i\left(\xi-\xi_{1}\right)} U_{1}\left(z_{1}\right) d \xi_{1},
$$

with $Q\left(z, z_{1}\right)$ the quantity defined in (19a). This is an integral equation for $U_{1}(z)$. By the familiar process of successive iteration it leads formally to the relation

with

$$
U_{1}(z)=Y_{1}(z)+\sum_{n=1}^{\infty} Y_{1}^{(n)}(z)
$$

$$
Y_{1}^{(n+1)^{\prime}}(z)=\frac{1}{\rho^{4 \mu}} \int_{\Gamma} Q\left(z, z_{1}\right) e^{-i\left(\xi-\xi_{1}\right)} Y_{1}^{(n)}\left(z_{1}\right) d \xi_{1},
$$

and $Y_{1}{ }^{(0)}(z) \equiv Y_{1}(z)$. Whenever it is uniformly convergent the relation (22) is a true formula for $U_{1}(z)$. It is to be shown that this is the case whenever $|\rho|$ is sufficiently large.

Consider the relation

$$
Y_{1}^{(n)}(z)=\frac{\xi^{4 n \mu+\mu+\beta}}{\rho^{4 n \mu}} O(1), \text { when }|\xi| \leqq N,
$$

with $O(1)$ representing a function which is bounded uniformly as to $n$. The relation is evidently satisfied when $n=0$ because of the formulas (9) and the boundedness of $|\xi|$. It may be shown as follows, however, that the validity of the relation for any $n$ implies it for the next larger value, so that by induction the relation will be established for all $n$.

Since the region $|\xi| \leqq N$ lies entirely in $R_{\xi}$ the path of integration in (23) may be taken straight. Then from the formulas (9) and (19a) it is seen that if $\beta \neq 0$ the character of the relation (23) is

$$
Y_{1}^{(n+1)}=\frac{1}{\rho^{4 \mu+4 n \mu}} \int_{\Gamma}\left\{\xi^{\mu+\beta} \xi_{1}^{4(n+1) \mu} O(1)+\xi^{\mu-\beta} \xi_{1}^{4(n+1) \mu+2 \beta} O(1)\right\} \frac{d \xi_{1}}{\xi_{1}} .
$$


But on setting $t=\xi_{1} / \xi$ this may be written

$$
Y_{1}^{(n+1)}=\frac{\xi^{4(n+1) \mu+\mu+\beta}}{\rho^{4(n+1) \mu}} \int_{0}^{1}\left\{O(1)+t^{2 \beta} O(1)\right\} t^{4(n+1) \mu-1} d t,
$$

and since $\mu$ is positive and the real part of $\beta$ is not negative this establishes (24) with $n$ replaced by $n+1$. If $\beta=0$ it is found in a similar way that

$$
Y_{1}^{(n+1)}=\frac{1}{\rho^{4 \mu+4 n \mu}} \int_{\Gamma} \xi^{\mu \xi_{1}^{4(n+1) \mu}}\left\{\log \xi_{1}-\log \xi\right\} O(1) \frac{d \xi_{1}}{\xi_{1}},
$$

and since this may be written

$$
Y_{1}^{(n+1)}=\frac{\xi^{4(n+1) \mu+\mu}}{\rho^{4(n+1) \mu}} \int_{0}^{1} t^{4(n+1) \mu-1} \log t O(1) d t,
$$

the conclusion again follows. The relation (24) is therefore generally valid.

It is evident now, in virtue of (24), that the uniform convergence of the series in (22) over the region $|\xi| \leqq N$ is assured when $|\rho|$ is sufficiently large. Since that is a blanket assumption for the entire discussion it follows that

i.e., more explicitly

$$
U_{1}(z)=Y_{1}(z)+\frac{\xi^{5 \mu+\beta}}{\rho^{4 \mu}} O(1), \text { when }|\xi| \leqq N,
$$

$$
u_{1}(z)=\Psi(z) \xi^{\mu+\beta}\left\{\xi^{-\beta} J_{\beta}(\xi)+\frac{\xi^{4 \mu} O(1)}{\rho^{4 \mu}}\right\} \text { when }|\xi| \leqq N .
$$

The formula (25) describes the solution $u_{1}(z)$ near the origin. Since it is of the order of $\xi^{\mu+\beta}$, i.e., of the order of $z^{1 / 2+A}$, while the exponents of the differential equation relative to $z=0$ are $\frac{1}{2} \pm A$, it is seen that when $\Re(A)>0$, $u_{1}(z)$ is the solution which vanishes at the origin to a higher order than any which is linearly independent of it. It may also be observed in connection with the formula (25) that the second term, i.e., the vague correction term, is of the order of $z^{2}$ as well as of the order of $\rho^{-4 \mu}$ relative to the explicit first term.

Finally, the substitution of the values $(25)$ and $\left(9^{\prime}\right)$ in the right-hand member of the derived relation $\left(20^{\prime}\right)$ yields for the integral concerned a form

$$
\frac{\xi^{3 \mu+\beta}}{\rho^{2 \mu}} \int_{0}^{1} t^{4 \mu-1}\left\{O(1)+t^{2 \beta} O(1)\right\} d t .
$$

Hence it may be concluded in precisely the manner above that

$$
u_{1}^{\prime}(z)=y_{1}^{\prime}(z)+\frac{\xi^{3 \mu+\beta} O(1)}{\rho^{2 \mu}}, \text { when }|\xi| \leqq N .
$$


This formula is precisely that which is obtained by a direct formal differentiation of (25).

6. The solution $u_{1}(z)$ when $|\xi|>N$. When $|\xi|$ is large the first or the second term of the formula $(17)$ for $y_{1}(z)$ is dominant according as $\Im(\xi)$ is negative or positive. The deductions for the solution $u_{1}(z)$ are to be based largely upon this formula and must, therefore, be appropriately adapted to the location of $\xi$. Since such adaptation extends merely to formal and rather obvious detail the explicit argument will be given only say for $\Im(\xi) \leqq 0$. The function $Y_{1}(z)$ defined in (21) is then of the structure $\xi^{\mu-1 / 2} O(1)$ when $|\xi|>N$, the same being true moreover of the functions defined by the formulas

$$
\begin{aligned}
& Y_{k, 1}(z) \equiv \frac{e^{-i \xi}}{\Psi(z)} y_{k, 1}(z), \\
& Y_{k, 2}(z) \equiv \frac{e^{i \xi}}{\Psi(z)} y_{k, 2}(z),
\end{aligned}
$$

provided $k$ is the index of the sub-region (13) in which $\xi$ is located, i.e.,

$$
Y_{k, j}(z)=\xi^{\mu-1 / 2} O(1), \quad j=1,2 \text {, when }|\xi|>N \text {, and } \xi \text { is in } \Xi^{(k)} \text {. }
$$

Let $\rho_{\mu}$ be defined appropriately to the value of $\mu$ in the manner

$$
\rho_{\mu}= \begin{cases}\rho, & \text { if } \mu>\frac{1}{4}, \\ \rho / \log \rho, & \text { if } \mu=\frac{1}{4}, \\ \rho^{4 \mu}, & \text { if } \mu<\frac{1}{4} .\end{cases}
$$

It evidently becomes infinite with $\rho$ in every case. When $n=0$ the relation

$$
Y_{1}^{(n)}(z)=\frac{\xi^{\mu-1 / 2}}{\rho_{\mu}^{n}} O(1), \text { when }|\xi|>N,
$$

is valid, as was observed above. It is to be shown on the basis of the relation (23) that it is valid for all $n$.

For the consideration of the integral in (23) let the $\Gamma$ curve of integration be sub-divided into the following component arcs: $\Gamma_{1}$, the arc on which $\left|\xi_{1}\right|$ $\leqq N ; \Gamma_{2}$, the arc on which $\left|\xi_{1}\right|>N$ but whose image in $R_{z}$ lies within a (any specified) finite portion of $R_{z} ; \Gamma_{3}$, the remaining arc if any. If $R_{z}$ is finite no arc $\Gamma_{3}$ need be considered.

* The formula (21) was in fact designed to produce this result. If $\xi$ is to be taken in an upper half-plane the formula (21) should be modified by replacing $-i$ by $i$, a change which in no way affects the reasoning in $\$ 5$. 
The relations (19b) and (10) may be used to show without difficulty that the following are equivalent formulas, i.e.,

(30) $Q\left(z, z_{1}\right) e^{-i\left(\xi-\xi_{1}\right)}=$

$$
\begin{aligned}
& \frac{i \Psi^{3}\left(z_{1}\right) \theta\left(\rho, z_{1}\right)}{2}\left\{Y_{k, 1}(z) y_{k, 2}\left(z_{1}\right)\right. \\
& \left.-Y_{k, 2}(z) y_{k, 1}\left(z_{1}\right) e^{-2 i \xi}\right\} e^{i \xi_{1} \xi_{1}^{2 \mu-1}}, \\
& \frac{i \Psi^{4}\left(z_{1}\right) \theta\left(\rho, z_{1}\right)}{2}\left\{Y_{k, 1}(z) Y_{k, 2}\left(z_{1}\right)\right. \\
& \left.-Y_{k, 2}(z) Y_{k, 1}\left(z_{1}\right) e^{-2 i\left(\xi-\xi_{1}\right)}\right\} \xi_{1}^{2 \mu-1}, \\
& \frac{i \theta\left(\rho, z_{1}\right)}{2 \rho^{1-4 \mu} \phi\left(z_{1}\right)}\left\{Y_{k, 1}(z) Y_{k, 2}\left(z_{1}\right)\right. \\
& \left.-Y_{k, 2}(z) Y_{k, 1}\left(z_{1}\right) e^{-2 i\left(\xi-\xi_{1}\right)}\right\} \xi_{1}^{1-2 \mu} \frac{d z_{1}}{d \xi_{1}} .
\end{aligned}
$$

They are to be used respectively for evaluating the integrations in (23) over the $\operatorname{arcs} \Gamma_{1}, \Gamma_{2}$ and $\Gamma_{3}$. The exponential factors are then bounded, since $\Im\left(\xi-\xi_{1}\right) \leqq 0$ whenever $\xi_{1}$ is on a $\Gamma$ curve which joins the origin with the point $\xi$. With the use of the formulas (27) when the variable is $z$; the formulas (12) and (24) when the variable is $z_{1}$ on $\Gamma_{1}$; and (27) and (29) when $z_{1}$ is on $\Gamma_{2}$ or $\Gamma_{3}$; it may then be shown that the relation (23) is structurally as follows:

$$
\begin{aligned}
Y_{1}^{(n+1)}= & \frac{\xi^{\mu-1 / 2}}{\rho_{\mu}^{n+1}}\left\{\left(\frac{\rho_{\mu}}{\rho^{4 \mu}}\right)^{n+1} \int_{\Gamma_{1}} O(1) d \xi_{1}+\frac{\rho_{\mu}}{\rho^{4 \mu}} \int_{\Gamma_{2}} \xi_{1}^{4 \mu-1} O(1) \frac{d \xi_{1}}{\xi_{1}}\right. \\
& \left.+\frac{\rho_{\mu}}{\rho} \int_{\Gamma_{3}} \frac{\theta\left(\rho, z_{1}\right)}{\phi\left(z_{1}\right)} O(1) d z_{1}\right\}
\end{aligned}
$$

In this the integral over $\Gamma_{1}$ and its coefficient are obviously bounded. The integral over $\Gamma_{2}$ is of the order of $\xi_{1}{ }^{4 \mu-1}, \log \xi_{1}$, or 1 , according as $4 \mu$ is greater than, equal to, or less than 1 . Since on this arc the value of $\xi_{1}$ is at most of the order of $\rho$, the order of the integral is seen to be the reciprocal of its coefficient so that the product of the two is bounded. The coefficient of the integral over $\Gamma_{3}$ is bounded. To insure finally the boundedness of this integral as well, the following is to be added as an hypothesis upon the given differential equation:

(v) In the region $R_{z}$ a relation

$$
\int\left|\frac{\theta(\rho, z)}{\phi(z)} d z\right|<M
$$

is satisfied by some constant $M$, uniformly with respect to all arcs of integration which are of the type $\Gamma$ for some admitted value of $\rho$, and on which $|z| \geqq N_{1}>0$. 
The form of $Y_{1}^{(n+1)}$ has thus been shown to be as given by (29), and the latter, therefore, to be valid for all $n$. The convergence of the formula (22) when $\rho$ is suitably large is therefore assured, whence it may be drawn that

i.e., that

$$
U_{1}(z)=Y_{1}(z)+\frac{\xi^{\mu-1 / 2}}{\rho_{\mu}} O(1), \text { when }|\xi|>N,
$$

$$
u_{1}(z)=y_{1}(z)+\Psi(z) \xi^{\mu-1 / 2} e^{i \xi} \frac{O(1)}{\rho_{\mu}} .
$$

This is the result obtained on the assumption that $\xi$ remains in a lower halfplane. The form correspondingly obtained when $\xi$ is in an upper half-plane differs from it only in that the factor $e^{i \xi}$ is replaced by $e^{-i \xi}$. For unrestricted variation of $\xi$ the result may, therefore, be expressed by the formula

(31) $u_{1}(z)=y_{1}(z)+\Psi(z) \xi^{\mu-1 / 2}\left\{\frac{e^{i \xi} O(1)+e^{-i \xi} O(1)}{\rho_{j}}\right\}$, when $|\xi|>N$.

A discussion entirely similar to that given but based upon the derived formula $\left(20^{\prime}\right)$ may be made to yield the representation of $u_{1}^{\prime}(z)$, or alternatively the direct differentiability of (31) may be justified. Upon substituting for $y_{1}(z)$ its forms $(17)$ and $\left(17^{\prime}\right)$, it is to be concluded that when $|\xi|>N$,

and

$$
\begin{aligned}
u_{1}(z)= & \Psi(z) \xi^{\mu-1 / 2}\left\{c_{1} e^{i \xi}\left[1+O\left(\frac{1}{\xi}\right)+O\left(\frac{1}{\rho_{\mu}}\right)\right]\right. \\
& \left.+c_{2} e^{-i \xi}\left[1+O\left(\frac{1}{\xi}\right)+O\left(\frac{1}{\rho_{\mu}}\right)\right]\right\},
\end{aligned}
$$

$$
\begin{aligned}
u_{1}^{\prime}(z)= & \frac{i \rho^{2 \mu} \xi^{-\mu+1 / 2}}{\Psi(z)}\left\{c_{1} e^{i \xi}\left[1+O\left(\frac{1}{\xi}\right)+O\left(\frac{1}{\rho_{\mu}}\right)\right]\right. \\
& \left.-c_{2} e^{-i \xi}\left[1+O\left(\frac{1}{\xi}\right)+O\left(\frac{1}{\rho_{\mu}}\right)\right]\right\},
\end{aligned}
$$

with the coefficients (18).

THEOREM 1. Under the hypotheses (i) to (v) the solution of the differential equation (2) with the exponent $\frac{1}{2}+A$ relative to the origin has the form (25), $\left(25^{\prime}\right)$ for values of $z$ such that $|\xi| \leqq N$, and the asymptotic forms (32), (32') with coefficients (18) when $|\xi|>N$.

7. The sub-dominant solutions. The results of the preceding section show that the solution $u_{1}(z)$ is of the dominant type for all admitted ranges of the variable. It is to be shown now that the differential equation admits 
also solutions which are of the sub-dominant form in appropriately associated domains. These solutions are analogous to the solutions $y_{k, j}(z)$ of the related equation, and will, of course, be linearly independent of $u_{1}(z)$.

The domain common to a pair of adjacent sub-regions (13) lies wholly in either an upper or a lower half-plane. Its boundary, a portion of the boundary of $R_{\xi}$, either contains a point to be denoted by $\xi_{M}$, at which $\Im(\xi)$ is numerically a maximum, or else $\Im(\xi)$ is unbounded, the domain extending to infinity. A distinction between these cases will be avoided by permitting $\xi_{M}$ to designate either the finite or the infinite point. There will be one such point for each pair of adjacent sub-regions (13). Inasmuch as these points are fixed relative to $R_{\xi}$ the image points $z_{M}$ in $R_{z}$ in general vary with the parameter. The following hypothesis (the final one to be made) therefore again concerns the configurative character of $R_{z}$ together with the admitted range of values $\rho$.

(vi) The region $R_{z}$ is such that for any (every) admitted value of $\rho$ each point $\xi_{M}$ may be connected with any (every) point of $R_{\xi}$ in its respective halfplane by a curve of the type $\Gamma$ lying entirely in $R_{\xi}$.

It will be evident that in virtue of the earlier hypothesis (iv) each point $\xi_{M}$ may be connected by a $\Gamma$ curve not merely with any point of its own halfplane as stated, but in fact with any point of the two sub-regions (13) within which it lies.

A specific point $\xi_{M}$ lies either above or below the axis of reals and some adaptation of the details to the case in point must be made. In principle, however, the argument is general and the explicit discussion will therefore be given only say for a case in which $\Im\left(\xi_{M}\right)<0$. The regions $\Xi^{(k)}$ in which $\xi_{M}$ is included are then given by $k=2 m-1$ and $k=2 m$ with a suitable integer $m$. The variable $\xi$ will be supposed to remain in these same sub-regions throughout the discussion of this section.

Let the formula (20) be written with the roles of $y(z), z_{0}$, and $u(z)$ taken by $y_{k, 2}(z), z_{M}$, and $u_{k, 2}(z)$, the path of integration being chosen as a $\Gamma$ curve. With the use of the abbreviation

$$
U_{k, 2}(z) \equiv \frac{e^{i \xi}}{\Psi(z)} u_{k, 2}(z),
$$

the relation may then be written

$$
\begin{aligned}
U_{2 m-1,2}(z) & \equiv U_{2 m, 2}(z), \\
U_{2 m, 2}(z) & \equiv Y_{2 m, 2}(z)+\frac{1}{\rho^{4 \mu}} \int_{\Gamma} Q\left(z, z_{1}\right) e^{i\left(\xi-\xi_{1}\right)} U_{2 m, 2}\left(z_{1}\right) d \xi_{1},
\end{aligned}
$$

and this by iteration leads to the formal relation 


$$
U_{2 m, 2}(z)=Y_{2 m, 2}(z)+\sum_{n=1}^{\infty} Y_{2 m, 2}^{(n)}(z)
$$

with

$$
Y_{2 m, 2}^{(n+1)}(z)=\frac{1}{\rho^{4 \mu}} \int_{\Gamma} Q\left(z, z_{1}\right) e^{i\left(\xi-\xi_{1}\right)} Y_{2 m, 2}^{(n)}\left(z_{1}\right) d \xi_{1},
$$

and $Y_{m, 2}^{(0)}(z) \equiv Y_{2 m, 2}(z)$. It is to be shown that for all $n$

$$
Y_{2 m, 2}^{(n)}(z)=\frac{\xi^{\mu-1 / 2}}{\rho_{\mu}^{n}} O(1), \text { when }|\xi|>N .
$$

This is a fact when $n=0$, since the relation is then included in (27).

When $\xi$ lies in the region $|\xi|>N$ the same may be assumed of the entire curve $\Gamma,{ }^{*}$ and the latter therefore consists of at most $\operatorname{arcs} \Gamma_{2}$ and $\Gamma_{3}$ as such were described in $\$ 6$. Upon these arcs the second and third of the formulas (30) multiplied by $e^{2 i\left(\xi-\xi_{1}\right)}$ may be respectively used to give the kernel of the formula (34). The exponentials involved are seen to be bounded since $\Im\left(\xi-\xi_{1}\right) \geqq 0$ when $\xi_{1}$ is on a $\Gamma$ curve joining $\xi$ with a point $\xi_{M}$ such as is being considered. If then the relation (35) is assumed to hold for any $n$, the structure of (34) is

$$
Y_{2 m, 2}^{(n+1)}=\frac{\xi^{\mu-1 / 2}}{\rho_{\mu}^{n+1}}\left\{\frac{\rho_{\mu}}{\rho} \int_{\Gamma_{2}} \frac{\theta\left(\rho, z_{1}\right)}{\phi\left(z_{1}\right)} O(1) d z_{1}+\frac{\rho_{\mu}}{\rho^{4 \mu}} \int_{\Gamma_{2}} \xi_{1}^{4 \mu-1} O(1) \frac{d \xi_{1}}{\xi_{1}}\right\}
$$

and the members within the brace are bounded as was found in $\$ 6$. The relation (35) is thus generally valid, the formula (33) accordingly convergent, and in consequence

Hence

$$
U_{2 m, 2}(z)=Y_{2 m, 2}(z)+\frac{\xi^{\mu-1 / 2}}{\rho_{\mu}} O(1), \text { when }|\xi|>N .
$$

$$
u_{2 m-1,2}(z) \equiv u_{2 m, 2}(z),
$$

$$
\begin{aligned}
& u_{2 m, 2}(z)=y_{2 m, 2}(z)+\Psi(z) \xi^{\mu-1 / 2} e^{i \xi} \frac{O(1)}{\rho_{\mu}}, \text { when }|\xi|>N, \\
& \text { and } \xi \text { is in } \Xi^{(2 m-1)} \text { or } \Xi^{(2 m)},
\end{aligned}
$$

and the formula may be shown to be differentiable.

* The distortion of the curve which may be necessary to circumvent the domain $|\xi| \leqq N$ is always slight and is readily seen to be negligible. 
When $\xi_{M}$ lies in an upper half-plane it is contained in a pair of sub-regions $\Xi^{(2 m)}, \Xi^{(2 m+1)}$. The discussion corresponding to that given above and based on the abbreviation

$$
U_{k, 1}(z) \equiv \frac{e^{-i \xi}}{\Psi(z)} u_{k, 1}(z)
$$

is found then to lead to the result

$$
u_{2 m+1,1}(z) \equiv u_{2 m, 1}(z) \text {, }
$$

$$
u_{2 m, 1}(z)=y_{2 m, 1}(z)+\Psi(z) \xi^{\mu-1 / 2} e^{i \xi} \frac{O(1)}{\rho_{\mu}}, \text { when }|\xi|>N,
$$

and $\xi$ is in $\Xi^{(2 m)}$ or $\Xi^{(2 m+1)}$.

On substituting the forms (14) it is thus established that when $|\xi|>N$

$$
\begin{aligned}
& u_{2 m, 1}(z)=\Psi(z) \xi^{\mu-1 / 2} e^{i \xi}\left\{1+O\left(\frac{1}{\xi}\right)+O\left(\frac{1}{\rho_{\mu}}\right)\right\}, \\
& u_{2 m, 1}^{\prime}(z)=\frac{i \rho^{2 \mu} \xi^{-\mu+1 / 2}}{\Psi(z)} e^{i \xi}\left\{1+O\left(\frac{1}{\xi}\right)+O\left(\frac{1}{\rho_{\mu}}\right)\right\},
\end{aligned}
$$

$$
\text { for } \xi \text { in } \Xi^{(2 m)} \text { and } \Xi^{(2 m+1)} \text {; }
$$

$$
\begin{aligned}
& u_{2 m, 2}(z)=\Psi(z) \xi^{\mu-1 / 2} e^{-i \xi}\left\{1+O\left(\frac{1}{\xi}\right)+O\left(\frac{1}{\rho_{\mu}}\right)\right\}, \\
& u_{2 m, 2}^{\prime}(z)=\frac{-i \rho^{2 \mu} \xi^{-\mu+1 / 2}}{\Psi(z)} e^{-i \xi}\left\{1+O\left(\frac{1}{\xi}\right)+O\left(\frac{1}{\rho_{\mu}}\right)\right\},
\end{aligned}
$$

for $\xi$ in $\Xi^{(2 m-1)}$ and $\Xi^{(2 m)}$.

8. The solutions $u_{k, j}(z)$ for unrestricted values of $z$. When $|\xi| \leqq N$ the path of integration in the formula (34) may be chosen as a $\Gamma$ curve from $\xi_{M}$ to the edge of the domain $|\xi| \leqq N$, and thence to the point $\xi$ as any ordinary curve on which $\left|\xi_{1}\right|$ decreases monotonically and $\arg \xi_{1}$ is bounded. The initial part will thus consist of at most arcs of type $\Gamma_{3}$ and $\Gamma_{2}$, and on these the equivalent forms

$$
Q\left(z, z_{1}\right) e^{i\left(\xi-\xi_{1}\right)}=\left\{\begin{array}{l}
\frac{i \theta\left(\rho, z_{1}\right) e^{i \xi}}{2 \rho^{1-4 \mu} \phi\left(z_{1}\right)}\left\{y_{k, 1}(z) Y_{k, 2}\left(z_{1}\right) e^{-2 i \xi_{1}}-y_{k, 2}(z) Y_{k, 1}\left(z_{1}\right)\right\} \xi_{1}^{1-2 \mu} \frac{d z_{1}}{d \xi_{1}}, \\
\frac{i \Psi^{4}\left(z_{1}\right) \theta\left(\rho, z_{1}\right) e^{i \xi}}{2 \Psi(z)}\left\{y_{k, 1}(z) Y_{k, 2}\left(z_{1}\right) e^{-2 i \xi_{1}}-y_{k, 2}(z) Y_{k, 1}\left(z_{1}\right)\right\} \xi_{1}{ }_{1}^{2 \mu-1}
\end{array}\right.
$$

may be respectively used in conjunction with the relations (35), (27) and (12). For the integration over the remaining arc, which will for convenience 
be denoted by $\Gamma_{1}$, the formula (19a) may be drawn on for $Q\left(z, z_{1}\right)$, and used in conjunction with (9). If it is assumed then that the relation

$$
Y_{2 m, 2}^{(n)}(z)=\left\{\begin{array}{ll}
\frac{\xi^{\mu-\beta}}{\rho_{\mu}^{n}} O(1), & \text { if } \beta \neq 0, \\
\frac{\xi^{\mu} \log \xi}{\rho_{\mu}^{n}} O(1), & \text { if } \beta=0,
\end{array} \text { when }|\xi| \leqq N,\right.
$$

holds for any specific value of $n$, it is found that when $\beta \neq 0$ (34) is of the form

$$
\begin{aligned}
Y_{2 m, 2}^{(n+1)}= & \frac{\xi^{\mu-\beta}}{\rho_{\mu}^{n+1}}\left\{\frac{\rho_{\mu}}{\rho} \int_{\Gamma_{3}} \frac{\theta\left(\rho, z_{1}\right)}{\phi\left(z_{1}\right)} O(1) d z_{1}+\frac{\rho_{\mu}}{\rho^{4 \mu}} \int_{\Gamma_{2}} \xi_{1}^{4 \mu-1} O(1) \frac{d \xi_{1}}{\xi_{1}}\right. \\
& \left.+\frac{\rho_{\mu}}{\rho^{4 \mu}} \int_{\Gamma_{1}}\left[\left(\frac{\xi}{\xi_{1}}\right)^{2 \beta} O(1)+O(1)\right] \xi_{1}^{4 \mu-1} d \xi_{1}\right\} .
\end{aligned}
$$

The formula when $\beta=0$ differs from this in details which will now be familiar. The terms involving the integrals over $\Gamma_{3}$ and $\Gamma_{2}$ are of forms which have previously been discussed, and found to be bounded. In the integral over $\Gamma_{1}$ the quantity $\left(\xi / \xi_{1}\right)$ never exceeds unity in numerical value and is of bounded argument, while $\Re(2 \beta) \geqq 0$, and as a result the term in question is seen to be likewise bounded. Hence the relation (38) remains valid when $n$ is replaced by $n+1$, and since it is evidently so when $n=0$ it is valid for all $n$.

From (38) and (33) it follows that the formula

$$
U_{2 m, j}(z)=Y_{2 m, j}(z)+ \begin{cases}\frac{\xi^{\mu-\beta}}{\rho_{\mu}} O(1), & \text { if } \beta \neq 0, \\ \frac{\xi^{\mu} \log \xi}{\rho_{\mu}} O(1), & \text { if } \beta=0,\end{cases}
$$

holds when $j=2$. An entirely similar discussion may be made to show that it holds also when $j=1$. The conclusion to be drawn is, therefore, that when $|\xi| \leqq N$ and $\arg z$ is bounded,

$$
u_{2 m, j}(z)=y_{2 m, j}(z)+ \begin{cases}\Psi(z) \xi^{\mu-\beta} \frac{O(1)}{\rho_{\mu}}, & \text { if } \beta \neq 0, \\ \Psi(z) \xi^{\mu} \log \xi \frac{O(1)}{\rho_{\mu}}, & \text { if } \beta=0 .\end{cases}
$$

The associated result 


$$
u_{2 m, j}^{\prime}(z)=y_{2 m, j}^{\prime}(z)+ \begin{cases}\frac{\xi^{-\mu-\beta}}{\Psi(z)} \frac{\rho^{2 \mu} O(1)}{\rho_{\mu}}, & \text { if } \beta \neq 0, \\ \frac{\xi^{-\mu} \log \xi}{\Psi(z)} \frac{\rho^{2 \mu} O(1)}{\rho_{\mu}}, & \text { if } \beta=0,\end{cases}
$$

is obtainable by the substitution of (39) into the derived relation $\left(20^{\prime}\right)$.

The formulas (39), $\left(39^{\prime}\right)$ give the descriptions of the solutions $u_{k, j}(z)$ for values of $z$ such that $|\xi| \leqq N$. It only remains, therefore, to consider the forms of these solutions when $|\xi|>N$ and $\xi$ is not in one of the specially associated sub-regions (13) indicated in the concluding formulas of $\$ 7$. In this connection the following considerations may be made.

Every value $\xi$ is included in some sub-region (13), and hence any given $\xi$ may be said to lie in $\Xi^{(h)}$ since this amounts merely to a specification of the index $h$. The associated solutions $u_{h, j}(z)$ are then of the forms which have been deduced above. In terms of them the solutions of any arbitrarily chosen pair, say $u_{k, j}(z)$, may be expressed linearly, i.e.,

$$
u_{k, j}(z) \equiv C_{1, j} u_{h, 1}(z)+C_{2, j} u_{h, 2}(z),
$$

with coefficients $C_{i, j}$ independent of $z$. The identities between the corresponding solutions of the related equation may be written

$$
y_{k, j}(z) \equiv C_{1, j}^{\prime} y_{h, 1}(z)+C_{2, j}^{\prime} y_{h, 2}(z) .
$$

Now when $|\xi| \leqq N$ the solutions involved in (40) are given by the formulas (39), and hence (40) is also expressible in the form

$$
y_{k, j}(z)+\Psi(z) \xi^{\mu-\beta} \frac{O(1)}{\rho_{\mu}} \equiv C_{1, j} y_{h, 1}(z)+C_{2, j} y_{h, 2}(z) .
$$

On subtracting (41) from this it is accordingly found that

$$
\left(C_{1, j}-C_{1, j}^{\prime}\right) y_{h, 1}(z)+\left(C_{2, j}-C_{2, j}^{\prime}\right) y_{h, 2}(z) \equiv \Psi(z) \xi^{\mu-\beta} \frac{O(1)}{\rho_{\mu}},
$$

a relation which in virtue of the forms (12) implies that

$$
C_{i, j}=C_{i, j}^{\prime}+\frac{O(1)}{\rho_{\mu}}, \quad i, j=1,2 .
$$

On inserting these evaluations in (40), however, and allowing $\xi$ to take large values in $\Xi^{(h)}$ the comparison of the right-hand members of (40) and (41) leads to the conclusion that 


$$
u_{k, j}(z)=y_{k, j}(z)+\Psi(z) \xi^{\mu-1 / 2}\left\{\frac{e^{i \xi} O(1)+e^{-i \xi} O(1)}{\rho_{\mu}}\right\}, \text { when }|\xi|>N .
$$

Since the index $h$ is not in evidence, this formula is valid for an arbitrary location of $\xi$ in the region $|\xi|>N$. Finally, if the forms (15) are inserted for the solutions $y_{k, j}$ the results are the following:

$$
\begin{aligned}
u_{2 m, 1}(z) \equiv & u_{2 m+1,1}(z), \quad u_{2 m-1,2}(z) \equiv u_{2 m, 2}(z), \\
u_{2 m, j}(z)= & \Psi(z) \xi^{\mu-1 / 2}\left\{e^{i \xi}\left[c_{j, 1}^{(m)}+O\left(\frac{1}{\xi}\right)+O\left(\frac{1}{\rho_{\mu}}\right)\right]\right. \\
& \left.+e^{-i \xi}\left[c_{j, 2}^{(m)}+O\left(\frac{1}{\xi}\right)+O\left(\frac{1}{\rho_{\mu}}\right)\right]\right\},
\end{aligned}
$$

with coefficients dependent upon the location of $\xi$ as given in (16). These formulas include the formulas (37). However, when they are applicable the latter are more precise, since in (43) the vanishing of the entire coefficient of an exponential cannot be directly inferred from the vanishing of the constant $c_{j, 1}^{(m)}$ involved, and the identification of a solution as of the sub-dominant type may thereby be made impossible.

THEOREM 2. Under the hypotheses (i) to (vi) the differential equation (2) admits of fundamental pairs of solutions $u_{k, j}(z), j=1,2 ; k=0, \pm 1, \pm 2, \cdots$, which are of the forms (39), (39') for values of $z$ such that $|\xi| \leqq N$, and for values of $z$ such that $|\xi|>N$ are generally of the forms (43) with the coefficients (16), and more specifically of the forms (37) in the sub-regions for which the latter are indicated to be valid.

UNIVERSITY OF WISCONSIN, Madison, WIs. 